\title{
THE RUDIN KERNEL AND THE EXTREMAL FUNCTIONS IN HARDY CLASSES
}

\author{
By Saburou Saitoh
}

1. In [8], we have been concerned with the Rudin kernel and some associated reproducing kernels on compact bordered Riemann surfaces. The Rudin kernel which is analytic on $\bar{S}$ (i.e. the closure of $S$ ) is characterized by the following reproducing property on a compact bordered Riemann surface $S$ : Let $H_{2}(S)$ be the class of analytic functions $f$ on $S$ such that $|f|^{2}$ has harmonic majorants on $S$. For fixed $x, t \in S$,

$$
f(x)=\frac{1}{2 \pi} \int_{\partial S} f(\tau) \overline{R_{t}(\tau, x)} \frac{\partial g(\tau, t)}{\partial \nu} d s_{\tau} \quad \text { for all } f \in H_{2}(S) .
$$

Here $\partial S$ is the relative boundary of $S, g(\tau, t)$ is the Green function of $S$ with pole at $t$ and the derivative is taken along the inner normal. Furthermore the adjoint $L$-kernel $\mathcal{L}_{t}(\tau, t)$ is characterized by the following relation:

$$
\overline{R_{t}(\tau, x)} \frac{\partial g(\tau, t)}{\partial \nu} d s_{\tau}=\frac{1}{i} \mathcal{L}_{t}(\tau, x) \quad \text { along } \quad \partial S
$$

Here $\mathcal{L}_{t}(\tau, x)$ is an analytic differential on $\bar{S}$ except for a simple pole at $x$ with residue 1. However as we have pointed out (cf. [8], Lemma 3.1), the Rudin kernel on a compact bordered Riemann surface does not characterize completely the Rudin kernel. In the present paper, we shall be concerned with some further properties of the Rudin kernel on an arbitrary Riemann surface and a general region in the plane. Let $S$ be an arbitrary open Riemann surface. For fixed $x, t(\in S)$, let $\left\{S_{n}\right\}_{n=0}^{\infty}\left(S_{0} \ni x, t\right)$ be a regular exhaustion of $S$. Let $R_{t}^{(n)}(\tau, x)$ denote the Rudin kernel of $S_{n}$ with respect to $t$ and $x$. Let $g_{n}(\tau, t)$ denote the Green function of $S_{n}$ with pole at $t$. Let $H_{p}(S)(p>0)$ be the class of analytic functions $f$ on $S$ for which $|f|^{p}$ has harmonic majorants on $S$. For any $f \in H_{p}(S)$, let $u_{f}$ denote the least harmonic majorant of $|f|^{p}$. Then we define $H_{p}$-norm on $S$ with respect to $t$ by $\|f\|_{S, p}^{t}=u_{f}(t)^{1 / p}$ for any fixed point $t$ on $S$.

The author wishes to express here his sincere thanks to Professor N. Suita for his encouragement and useful criticisms.

2. At first, the following theorem is fundamental:

Received December 17, 1971. 
THEOREM 2.1. The sequence of the Rudin kernels $\left\{R_{t}^{(n)}(\tau, x)\right\}_{n=0}^{\infty}$ converges uniformly to an $H_{2}(S)$-function $R_{t}(\tau, x)$ on an arbitrary compact subset of $S$. Further it also converges to $R_{t}(\tau, x)$ in $H_{2}$-norm. $R_{t}(\tau, x)$ is determined uniquely and independently of the choice of regular exhaustions $\left\{S_{n}\right\}$. Moreover the kernel is characterized by the following reproducing property:

$$
f(x)=\lim _{n \rightarrow \infty} \frac{1}{2 \pi} \int_{\partial S_{n}} f(\tau) \overline{R_{t}(\tau, x)} \frac{\partial g_{n}(\tau, t)}{\partial \nu} d s_{\tau} \quad \text { for all } f \in H_{2}(S)
$$

Proof. From the reproducing property, we have

$$
R_{t}^{(n)}(\tau, x)=\frac{1}{2 \pi} \int_{\partial S_{n}} R_{t}^{(n)}(\tilde{\tau}, x) \overline{R_{t}^{(n)}(\tilde{\tau}, \tau)} \frac{\partial g_{n}(\tilde{\tau}, t)}{\partial \nu} d s_{\tilde{\tau}}
$$

Hence we have

$$
\left|R_{t}^{(n)}(\tau, x)\right|^{2} \leqq R_{t}^{(n)}(x, x) R_{t}^{(n)}(\tau, \tau)
$$

Since $R_{t}^{(n)}(x, x)$ decreases with respect to $n$ [8], $\left\{R_{t}^{(n)}(\tau, x)\right\}$ is locally uniformly bounded and therefore it forms a normal family. By passing to a subsequence if necessary, we may assume that $\left\{R_{t}^{(n)}(\tau, x)\right\}$ itself converges uniformly on compact subsets of $S$ to an analytic function $R_{t}(\tau, x)$. At first, we shall prove $R_{t}(\tau, x) \in H_{2}(S)$.

For any $m, n$ such that $S_{n} \supset S_{m}$, we have

$$
\begin{aligned}
& \frac{1}{2 \pi} \int_{\partial S_{m}}\left|R_{t}(\tau, x)\right|^{2} \frac{\partial g_{m}(\tau, t)}{\partial \nu} d s_{\tau} \\
= & \lim _{n \rightarrow \infty} \frac{1}{2 \pi} \int_{\partial S_{m}}\left|R_{t}^{(n)}(\tau, x)\right|^{2} \frac{\partial g_{m}(\tau, t)}{\partial \nu} d s_{\tau} \\
\leqq & \lim _{n \rightarrow \infty} \frac{1}{2 \pi} \int_{\partial S_{n}}\left|R_{t}^{(n)}(\tau, x)\right|^{2} \frac{\partial g_{n}(\tau, t)}{\partial \nu} d s_{\tau} \\
= & \lim _{n \rightarrow \infty} R_{t}^{(n)}(x, x)=R_{t}(x, x) .
\end{aligned}
$$

On letting $m$ tend to infinity, we have $\left\|R_{t}(\tau, x)\right\|_{S, 2}^{t} \leqq R_{t}(x, x)^{1 / 2}$. More precisely, we shall show $\left\|R_{t}(\tau, x)\right\|_{S, 2}^{t}=R_{t}(x, x)^{1 / 2}$.

Because $R_{t}^{(n)}(\tau, x) / R_{t}^{(n)}(x, x)$ is the unique extremal function minimizing $H_{2^{-}}$ norms among the functions $f \in H_{2}\left(S_{n}\right)$ with $f(x)=1$ [8], we obtain

$$
\frac{1}{2 \pi} \int_{\partial S_{n}}\left|\frac{R_{t}^{(n)}(\tau, x)}{R_{t}^{(n)}(x, x)}\right|^{2} \frac{\partial g_{n}(\tau, t)}{\partial \nu} d s_{\tau} \leqq \frac{1}{2 \pi} \int_{\partial S_{n}}\left|\frac{R_{t}(\tau, x)}{R_{t}(x, x)}\right|^{2} \frac{\partial g_{n}(\tau, t)}{\partial \nu} d s_{\tau} .
$$

(Note that $R_{t}(x, x)=\lim _{n \rightarrow \infty} R_{t}^{(n)}(x, x) \geqq 1$.) 
Hence

$$
\frac{R_{t}(x, x)^{2}}{R_{t}^{(n)}(x, x)} \leqq \frac{1}{2 \pi} \int_{\partial S_{n}}\left|R_{t}(\tau, x)\right|^{2} \frac{\partial g_{n}(\tau, t)}{\partial \nu} d s_{\tau} .
$$

Letting $n$ tend to infinity, we have $R_{t}(x, x)^{1 / 2} \leqq\left\|R_{t}(\tau, x)\right\|_{S, 2}^{t}$.

Next, we shall see that $R_{t}(\tau, x)$ has the reproducing property on $S$. For any $f \in H_{2}(S)$,

$$
\begin{aligned}
& \left|f(x)-\frac{1}{2 \pi} \int_{\partial S_{n}} f(\tau) \overline{R_{t}(\tau, x)} \frac{\partial g_{n}(\tau, t)}{\partial \nu} d s_{\tau}\right| \\
= & \left|\frac{1}{2 \pi} \int_{\partial S_{n}} f(\tau) \overline{\left[R_{t}^{(n)}(\tau, x)-R_{t}(\tau, x)\right]} \frac{\partial g_{n}(\tau, t)}{\partial \nu} d s_{\tau}\right| \\
\leqq & \|f\|_{S_{n}, 2}^{t}\left(\frac{1}{2 \pi} \int_{\partial S_{n}}\left|R_{t}^{(n)}(\tau, x)-R_{t}(\tau, x)\right|^{2} \frac{\partial g_{n}(\tau, t)}{d \nu} d s_{\tau}\right)^{1 / 2} \\
\leqq & \|f\|_{S, 2}^{t}\left[R_{t}^{(n)}(x, x)-2 R_{t}(x, x)+\left(\left\|R_{t}(\tau, x)\right\|_{S_{n}, 2}^{t}\right)^{2}\right]^{1 / 2}
\end{aligned}
$$

On letting $n$ tend to infinity, we see that $R_{t}(\tau, x)$ has the reproducing property for $\left\{S_{n}\right\}$. Because the $H_{2}$-function which has this property is determined uniquely, $R_{t}(\tau, x)$ is determined uniquely and for any regular exhaustion $\left\{S_{n}\right\}$ (hence in fact for any exhaustion in the sense of Lemma 3.1) $R_{t}(\tau, x)$ has the reproducing property on $S$.

From this theorem, we have a formula of Poisson-Jensen type on an arbitrary open Riemann surface by making use of $R_{t}(t, x)=1$ and the identity

$$
f(t)=\lim _{n \rightarrow \infty} \frac{1}{2 \pi} \int_{\partial S_{n}} f(\tau) R_{t}(\tau, x) \frac{\partial g_{n}(\tau, t)}{\partial \nu} d s_{\tau}, \quad \text { for all } f \in H_{2}(S) .
$$

Corollary 2.1.

$$
f(x)=-\overline{f(t)}+\lim _{n \rightarrow \infty} \frac{1}{\pi} \int_{\partial S_{n}} \operatorname{Re}[f(\tau)] \overline{R_{t}(\tau, x)} \frac{\partial g_{n}(\tau, t)}{d \nu} d s_{\tau},
$$

for all $f \in H_{2}(S)$.

Corollary 2.2. $f(x)=f(t)$ for all $f \in H_{2}(S)$ if and only if $R_{t}(x, x)=1$. Especially in a plane region $G$, for some $x, t \in G, x \neq t$ ) (and hence for all $x, t \in G, x \neq t$ ), $R_{t}(x, x)=1$ if and only if $G \in O_{H_{2}}$ (i.e. the class of Riemann surfaces for which $\mathrm{H}_{2}(\mathrm{~S})$ contains only constant members).

3. Let $H_{p}^{t}(S)$ denote the linear space of all $f \in H_{p}(S)$ with finite norm at $t \in S$. Let $H_{p}^{t 1}(S)$ be the unit ball $\|f\|_{s, p}^{t} \leqq 1$. Now we consider the extremal functions $f_{p}^{*}(\tau ; x, t)$ which maximize $f(x)$ in the subfamily of $H_{p}^{t 1}(S)$ satisfying $f(x) \geqq 0(p \geqq 1)$ (cf. [8]). If $p>1$, by virtue of the uniform convexity property and the compactness, we have the following lemma easily. 
LeMma 3.1. The extremal functions $f_{p}^{*}(\tau ; x, t)(p>1)$ exist uniquely. Let $\left\{S_{m}\right\}$ be a sequence of arbitrary domains containing $x$ and $t$ which exhausts $S$ from within. Then the sequence of the extremal functions $f_{p, m}^{*}(\tau ; x, t)$ which correspond to $\left\{S_{m}\right\}$ converges uniformly on compact subsets of $S$ to $f_{p}^{*}(\tau ; x, t)$. In particular, we have

(1) $f_{p}^{*}(x ; x, t)=1$ if and only if $f_{p}^{*}(\tau ; x, t) \equiv 1$.

(2) $f_{p}^{*}(\tau ; t, t) \equiv 1$.

(3) $f_{2}^{*}(\tau ; x, t)=R_{t}(\tau, x) / R_{t}(x, x)^{1 / 2}$.

In addition, (1) and (2) are valid for $f_{1}^{*}(\tau ; x, t)$.

Now, we state Theorem 3.1 as a generalization of Corollary 2.2 which is a direct result from Lemma 3.1 and the next lemma:

LEMMA 3.2.

$$
f(x)=f_{p}^{*}(x ; x, t) \lim _{n \rightarrow \infty} \frac{1}{2 \pi} \int_{\partial S_{n}} f(\tau) \frac{\left|f_{p}^{*}(\tau ; x, t)\right|^{p}}{f_{p}^{*}(\tau ; x, t)} \frac{\partial g_{n}(\tau, t)}{\partial \nu} d s_{\tau},
$$

for all $f \in H_{p}(S)(p \geqq 1)$.

Theorem 3.1. $f(x)=f(t)$ for all $f \in H_{p}(S)$ if and only if $f_{p}^{*}(x ; x, t)=1(p \geqq 1)$. Especially in a plane region $G$, for some $x, t(\epsilon G, x \neq t)$ (hence for all $x, t \in G, x \neq t$ ), $f_{p}^{*}(x ; x, t)=1$ if and only if $G \in O_{H_{p}}$.

Proof of lemma 3.2. We set $\hat{f}_{p}(\tau ; x, t)=f_{p}^{*}(\tau ; x, t) / f_{p}^{*}(x ; x, t)$, then $\hat{f}_{p}$ are the extremal functions which minimize $H_{p}$-norms in the subfamily of $H_{p}(S)$ satisfying $f(x)=1$. Consequently for any complex number $\lambda$ and $f \in H_{p}(S)$, we have

$$
\begin{aligned}
& \lim _{n \rightarrow \infty} \frac{1}{2 \pi} \int_{\partial S_{n}}\left|\hat{f}_{p}(\tau ; x, t)\right|^{p} \frac{\partial g_{n}(\tau, t)}{\partial \nu} d s_{\tau} \\
\leqq & \lim _{n \rightarrow \infty} \frac{1}{2 \pi} \int_{\partial S_{n}}\left|\hat{f}_{p}(\tau ; x, t)+\lambda(f(\tau)-f(x))\right|^{p} \frac{\partial g_{n}(\tau, t)}{\partial \nu} d s_{\tau} .
\end{aligned}
$$

By using a natural norm preserving isomorphism between $H_{p}(S)$ and the closed subspace of $H_{p}(\{|z|<1\})$ (cf. [7], p. 179), and from the arbitrariness of $\lambda$, we have

$$
\lim _{n \rightarrow \infty} \frac{1}{2 \pi} \int_{\partial S_{n}}(f(\tau)-f(x)) \frac{\left|\hat{f}_{p}(\tau ; x, t)\right|^{p}}{\hat{f}_{p}(\tau ; x, t)} \frac{\partial g_{n}(\tau, t)}{\partial \nu} d s_{\tau}=0
$$

Hence

$$
f(x)=\frac{1}{\left(\left\|\hat{f}_{p}\right\|_{S, p}^{t}\right)^{p}} \lim _{n \rightarrow \infty} \frac{1}{2 \pi} \int_{\partial S_{n}} f(\tau) \frac{\left|\hat{f}_{p}(\tau ; x, t)\right|^{p}}{\hat{f}_{p}(\tau ; x, t)} \frac{\partial g_{n}(\tau, t)}{\partial \nu} d s_{\tau} .
$$

Thus we have completed the proof of the lemma. 
4. Next in connection with Theorem 3.1, we assert the existence of a non$O_{H_{p}}$ Riemann surface on which $f(x)=f(t) \quad(x \neq t)$ for all $f \in H_{p}(S) \quad(p>0)$. The idea of this construction lies in Myrberg [6]. We introduce two copies $S_{1}, S_{2}$ of $\{z|| z \mid>1\}$ and distinguish the segments $[2 n, 2 n+1](n=1,2, \cdots)$. We construct the desired surface $S$ by joining $S_{1}$ to $S_{2}$ along their common distinguished slits in the usual manner. Let $\{x, t\}$ be the preimage of $z(|z|>1)$ with respect to the projection map. Then we see that $S \notin O_{H_{p}}(p>0)$ and $f(x)=f(t)$ for all $f \in H_{p}(S)$ (cf. [5] pp. 36-37).

5. Let $E$ be a compact subset of $S$ such that $S-E$ is connected. Then we shall investigate a property of $E$ for which $R_{t}^{(S)}(x, x)=R_{t}^{(S-E)}(x, x)(\neq 1, x \neq t$ and $x, t \in S-E)$ is valid. In the cases of the Szegö kernel and the Bergman kernel (with respect to the class of single-valued, square integrable, analytic functions having a single-valued indefinite integral) in the plane region, it is well known that such a case happens if and only if the analytic capacity of $E$ is zero and $E$ is a $N_{D}$-null set respectively (cf. [1], [4]). In our case, the condition of this type is quite different from theirs. We obtain such a condition from the following theorem by making use of the identity $f_{2}^{*}(\tau ; x, t) \equiv R_{t}(\tau, x) / R_{t}(x, x)^{1 / 2}$.

THEOREM 5.1. Any non-constant $H_{p}(S-E)$-function $f$ can be continued analytically to $S$ in the following way:

$$
\|f\|_{S, p}^{t}=\|f\|_{S-E, p}^{t}
$$

if and only if (logarithmic) Cap $E=0$. Here the norms are considered with respect to an arbitrary point $t(\in S-E)$ (hence all $t \in S-E)$.

Corollary 5.1. For some $x, t(\epsilon S-E, x \neq t$ ) (hence for all $x, t \in S-E, x \neq t$ ), $R_{t}^{(S)}(x, x)=R_{t}^{(S-E)}(x, x)(\neq 1)$ if and only if Cap $E=0$.

Proof of theorem 5.1. At first, we assume that a non-constant $H_{p}(\hat{S})(\hat{S} \equiv S-E)$ function $f$ can be continued analytically to $S$ in the following way: $\|f\|_{\hat{S}, p}^{t}=\|f\|_{S, p}^{t}$. Let $u$ and $\hat{u}$ be the least harmonic majorants of $|f|^{p}$ on $S$ and $\hat{S}$, respectively. Then we have $\hat{u}(\tau) \leqq u(\tau)$ on $\hat{S}$. From the assumption, we have $\hat{u}(t)=u(t)$ for $t(\epsilon \hat{S})$. Hence from the maximum principle we have $\hat{u}(\tau) \equiv u(\tau)$ on $\hat{S}$. Let $\left\{S_{m}\right\}_{m=0}^{\infty}$ be a regular exhaustion of $\hat{S}$ and we define $S_{m, n}$ as the component of $S_{m} \cap S_{n}$ which contains $t$. Then $\hat{u}(\tau)$ may be represented as follows:

$$
\hat{u}(\tau)=\lim _{\substack{n \rightarrow \infty \\ m \rightarrow \infty}} \frac{1}{2 \pi} \int_{\partial S_{m, n}}|f(\tilde{\tau})|^{p} \frac{\partial g_{m, n}(\tilde{\tau}, \tau)}{\partial \nu} d s_{\tilde{\tau}}, \quad \text { for all } \tau \in \hat{S} .
$$

If Cap $E \neq 0$, at least $E$ contains a regular boundary point $a_{0}$ in the sense of the Dirichlet problem (cf. [2]). Because $|f|^{p}$ is continuous on $E$, we have

$$
\lim _{\tau \rightarrow a_{0}} \hat{u}(\tau)=\hat{u}\left(a_{0}\right)=u\left(a_{0}\right)=\left|f\left(a_{0}\right)\right|^{p} .
$$


From the maximum principle, we have $|f(\tau)|^{p} \equiv u(\tau)$, which is absured.

On the other hand, if Cap $E=0$, it is well known that $H_{p}(\hat{S})$-functions can be continued analytically to $S$ [7]. It is readily verified that this extension has the desired property.

6. In this section, we shall consider the relations between the extremal functions $f_{p}^{*}(\tau ; x, t)$ and the Rudin kernel $R_{t}(\tau, x)$ on $S$. If $R_{t}(\tau, x)$ has no zeros and $R_{t}(\tau, x)^{2 / p}$ becomes a single-valued analytic function in $S$, then in some cases these relations are very simple. Here the branch of $R_{t}(\tau, x)^{2 / p}$ is determined by $R_{t}(x, x)^{2 / p} \geqq 1$ in the sequel. In addition, these relations should be compared with the case of $E_{p}$-classes in the plane region which was considered by Havinson [4].

THEOREM 6.1. (I) $2>p \geqq 1$ : For all $n>n_{0}$ (for some $n_{0}$ ), if $R_{t}^{(n)}(\tau, x)$ has no zeros in $S_{n}$ and $R_{t}(\tau, x)^{2 / p}$ is a single-valued analytic function in $S$, then the following relation (6.1) is valid.

(II) p>2: If $R_{t}(\tau, x)$ has no zeros and $R_{t}(\tau, x)^{2 / p}$ is a single-valued analytic function in $S$, then (6.1) is valid.

$$
f(x)=R_{t}(x, x)^{2 / p-1} \lim _{n \rightarrow \infty} \frac{1}{2 \pi} \int_{\partial S_{n}} f(\tau) \frac{\left|R_{t}(\tau, x)\right|^{2}}{R_{t}(\tau, x)^{2 / p}} \frac{\partial g_{n}(\tau, t)}{\partial \nu} d s_{\tau}
$$

for all $f \in H_{p}(S)$.

In particular, from (6.1) we have

$$
f_{p}^{*}(\tau ; x, t)=\frac{R_{t}(\tau, x)^{2 / p}}{R_{t}(x, x)^{1 / p}}, \quad \text { and } \quad f_{p}^{*}(x ; x, t) f_{p}^{*}(t ; x, t)=1 .
$$

Proof. In the case (I), we assume that $R_{t}^{(n)}(\tau, x)$ has no zeros in $S_{n}$ for all $n>n_{0}$. For any fixed $S_{n}$, we set

$$
\begin{gathered}
\tilde{f}_{1, n}(\tau ; x, t)=\frac{R_{t}^{(n)}(\tau, x)^{2}}{R_{t}^{(n)}(x, x)}\left(\epsilon H_{1}\left(S_{n}\right)\right), \quad \text { and } \\
\tilde{h}_{1, n}(\tau ; x, t)=\frac{1}{i d W_{n}(\tau, t)} \cdot \frac{1}{i} \cdot R_{t}^{(n)}(x, x) \cdot \frac{R_{t}^{(n)}(\tau, x) \mathcal{L}_{t}^{(n)}(\tau, x)}{R_{t}^{(n)}(\tau, x)^{2}} .
\end{gathered}
$$

Here $W_{n}(\tau, t)$ is defined by $g_{n}(\tau, t)+i g_{n}^{*}(\tau, t)$, where $g_{n}^{*}$ is the conjugate harmonic function of $g_{n}(\tau, t)$. From (1.1), we have

$$
\begin{aligned}
\tilde{f}_{1, n}(\tau ; x, t) \tilde{h}_{1, n}(\tau)= & \tilde{f}_{1, n}(x ; x, t)\left|\tilde{f}_{1, n}(\tau ; x, t)\right| \text { on } \partial S_{n}, \quad \text { and } \\
& \left\|\tilde{f}_{1, n}(\tau ; x, t)\right\|_{s_{n}, 1}^{t}=1 .
\end{aligned}
$$

From Theorem 3.1 in [8], we have

$$
\tilde{f}_{1, n}(\tau ; x, t) \equiv f_{1, n}^{*}(\tau ; x, t) \quad \text { and } \quad \tilde{h}_{1, n}(\tau) \equiv h_{1, n}(\tau) .
$$

Here $f_{1, n}^{*}$ and $h_{1, n}$ are the extremal functions on $S_{n}$ which we have stated there, Hence we have 


$$
f_{1, n}^{*}(\tau ; x, t)=\frac{R_{t}^{(n)}(\tau, x)^{2}}{R_{t}^{(n)}(x, x)}
$$

On letting $n$ tend to infinity, we have $f_{1}^{*}(\tau ; x, t)=R_{t}(\tau, x)^{2} / R_{t}(x, x)$, and therefore from Lemma 3.2 we have

$$
f(x)=R_{t}(x, x) \cdot \lim _{n \rightarrow \infty} \frac{1}{2 \pi} \int_{\partial S_{n}} f(\tau) \frac{\overline{R_{t}(\tau, x)}}{R_{t}(\tau, x)} \frac{\partial g_{n}(\tau, t)}{\partial \nu} d s_{\tau}, \quad \text { for all } f \in H_{1}(S) .
$$

Next we note that for $p \geqq 1$,

$$
\left|f(\tau) R_{t}(\tau, x)^{2(1-1 / p)}\right| \leqq \frac{1}{p}|f(\tau)|^{p}+\frac{p-1}{p}\left|R_{t}(\tau, x)\right|^{2}
$$

and therefore

$$
f(\tau) R_{t}(\tau, x)^{2(1-1 / p)} \in H_{1}(S) \quad \text { for all } f \in H_{p}(S)(p \geqq 1) .
$$

Hence we have

$$
f(x)=R_{t}(x, x)^{2 / p-1} \lim _{n \rightarrow \infty} \frac{1}{2 \pi} \int_{\partial S_{n}} f(\tau) R_{t}(\tau, x)^{2(1-1 / p)} \frac{\overline{R_{t}(\tau, x)}}{R_{t}(\tau, x)} \frac{\partial g_{n}(\tau, t)}{\partial \nu} d s_{\tau},
$$

for all $f \in H_{p}(S)(p \geqq 1)$, and therefore we obtain (6.1) for $p \geqq 1$.

At last, as to (II), we have

$$
f(\tau) R_{t}(\tau, x)^{(1-2 / p)} \in H_{2}(S), \quad \text { for all } f \in H_{p}(S)(p \geqq 2),
$$

and from Theorem 2.1, we obtain (6.1), similarly.

In the case of a simply connected region, we see that all assumptions of Theorem 6.1 are satisfied. More generally, in the case of a finitely connected plane region, we have the following theorem:

Theorem 6.2. Let $G$ be a finitely connected plane region. For fixed $t(\in G)$, there exists a neighborhood $U(t)$ of $t$ such that $R_{t}(\tau, x)(x \in U(t))$ has no zeros and $R_{t}(\tau, x)^{2 / p}(p>0)$ becomes a single-valued analytic function in $G$. In addition, in this case for $p=1$ (and therefore for $p \geqq 1$ ) the formula (6.1) is valid.

Proof. Without loss of generality, we may assume that $G$ is a regular region. Let $U_{0}(t)$ be a neighborhood of $t$ such that $t \in U_{0}(t) \subset \overline{U_{0}(t)} \subset G$. In particular, $R_{t}(z, x)\left(z \in \partial G, x \in \overline{U_{0}(t)}\right)$ is a continuous function for two variables $z$ and $x$ and therefore it is uniformly continuous on $\partial G \times \overline{U_{0}(t)}$. Hence for one, there exists $\delta>0$ such that

$$
\left|R_{t}(z, x)-R_{t}\left(z_{1}, x_{1}\right)\right|<1 \text { for all }\left|z-z_{1}\right|+\left|x-x_{1}\right|<\delta\left(z, z_{1} \in \partial G ; x, x_{1} \in \overline{U_{0}(t)}\right) \text {. }
$$

On setting $z_{1}=z$ and $x_{1}=t$, then from $R_{t}(z, t) \equiv 1$ (as we see from Theorem 2.1), we have 


$$
\left|R_{t}(z, x)-1\right|<1 \text {, for all } z \in \partial G \text { and all } x \in U_{0}(t) \text { such that }|x-t|<\delta \text {. }
$$

Hence we have a desired neighborhood $U(t)$ :

$$
U(t)=\{z|| z-t \mid<\delta\} \cap U_{0}(t) .
$$

From the conformal invariance of the $H_{p}$-norms and the proof of Theorem 6.1, we see that (6.1) is valid for $p=1$ in these cases.

Here we remark that in the case of infinitely connected plane region, Theorem 6.1 is no more valid in general. In fact, Hejhal [3] have pointed out the existence of a region $G$ such that $G \in O_{H_{2}}$ and $G \notin O_{H_{1}}$. From Theorems 3.1 and 6.1 , we shall see that for this region $G$, Theorem 6.1 is not valid. Further we note similarly that in the Case (I), the assumption " $R_{t}^{(n)}(\tau, x)$ (for all $n>n_{0}$ ) has no zeros in $S_{n}$ " can not be substituted for " $R_{t}(\tau, x)$ has no zeros in $S$ " in general. Next for a regular region $G$, let $F_{p}^{(N)}(z ; x, t)$ be the extremal functions which maximize $\left[d^{N} f(z) / d z^{N}\right]_{z=x}(N \geqq 1)$ in the subfamily of $H_{p}^{t 1}(G)$ satisfying $\left[d^{N} f(z) / d z^{N}\right]_{z=x} \geqq 0(p \geqq 1)$.

It is easy to see that

$$
F_{2}^{(N)}(z ; x, t)=\left[\frac{\partial^{N} R_{t}(z, y)}{\partial \bar{y}^{N}}\right]_{y=x} /\left[\frac{\partial^{2 N} R_{t}(z, y)}{\partial z^{N} \partial \bar{y}^{N}}\right]_{\substack{z=x \\ y=x}}^{1 / 2}, \quad \text { and } \quad F_{2}^{(N)}(t ; x, t)=0 .
$$

Therefore we note that for these extremal functions the circumstances as Theorem 6.1 does not happen. In addition, in connection with $F_{2}^{(N)}(t ; x, t)=0$, we see that $F_{p}^{(N)}(t ; x, t)=0(p \geqq 1, p \neq 2, N \geqq 1)$ is not valid in general.

7. Let $G$ be an $n$-ply connected regular region in the plane. It is wellknown as a very important property that the Szegö kernel and the Bergman kernel (which we have stated in No. 5) on $G$ have $n-1$ and $2 n-2$ zeros in $G$, respectively ([4], [9]). In this section, we shall consider the problem of the existence of zeros of the Rudin kernel. Unfortunately the total number of zeros depends on $G, x$, and $t$. We shall see these circumstances for the doubly connected plane region in detail. Without loss of generality, we may assume that $A$ is an annulus such that $\{z|r<| z \mid<1\}$. Let $f_{1}^{*}(z ; x, t)$ and $h_{1}(z)$ be the extremal functions which we have considered in ([8], Theorem 3.1). Then we have

$$
f_{1}^{*}(z ; x, t) h_{1}(z)=f_{1}^{*}(x ; x, t)\left|f_{1}^{*}(z ; x, t)\right| \quad \text { on } \partial A \text {. }
$$

Here $h_{1}(z)$ is the unique extremal function which minimizes ess. sup $|h(z)|(z \in \partial A)$ in the class of meromorphic functions such that

$$
\left\{\frac{1}{i W^{\prime}(z, t)}\left(\frac{1}{i(z-x)}+f(z)\right) \mid \text { for all } f \in H_{1}(A)\right\} \text {. }
$$

Since $h_{1}(z)$ is analytic on $\bar{A}$ and has at most one simple zero in $A$ except for $t$ (as we see from (7.1) by the argument principle), $h_{1}(z) / f_{1}^{*}(x ; x, t)$ is characterized 
as the unique extremal function which maximizes $\left|\operatorname{Res}_{z=x} h(z)\right|$ in the class of $h$ which satisfies the following conditions:

(1) $h(z)=\frac{c}{i W^{\prime}(z, t)}\left(\frac{1}{i(z-x)}+f(z)\right)$,

where $f(z)$ is an analytic function on $\bar{A}$ and $c$ is a real number.

(2) $|h(z)|=1$ on $\partial A$.

(3) $h(z)$ has at most one simple zero $\alpha$ in $A$ except for $t$.

Here if $x$ coincides with the critical point $t_{1}$ of $g(z, t), \operatorname{Res}_{z=x} h(z)$ is substituted for the coefficient of $1 /(z-x)^{2}$. Therefore for an admissible function $h$, we may represent the extremal property as follows:

$$
\log |h(z)|=g(z, x)-g(z, t)+g\left(z, t_{1}\right)-\delta g(z, \alpha),
$$

for some $\alpha \in A$ and $\delta=0$ or 1 .

$$
\omega_{1}(x)-\omega_{1}(t)+\omega_{1}\left(t_{1}\right)-\delta \omega_{1}(\alpha)=n_{1} \text { (integer). }
$$

Here $\omega_{1}(z)$ is the harmonic measure of the inner boundary $C_{1}$ of $A$.

$$
\begin{aligned}
\log \left|\operatorname{Res}_{z=x} h(z)\right| & =u(x)-g(x, t)+g\left(x, t_{1}\right)-\delta g(x, \alpha) \\
& =u(x)-g(t, x)+g\left(t_{1}, x\right)-\delta g(\alpha, x) .
\end{aligned}
$$

Here $u(z)$ is defined by $g(z, x)+\log |z-x|$. (7.3) represents the condition for $h(z)$ to become a single-valued function. More precisely, $n_{1}$ may be represented as follows:

$$
n_{1}=\frac{1}{2 \pi} \int_{C_{1}} d \arg h(z)=-\frac{1}{2 \pi} \int_{C_{1}} d \arg f_{1}^{*}(z ; x, t) \quad\left(-1 \leqq n_{1} \leqq 1\right) .
$$

Hence the zero $\alpha$ of $h_{1}(z) / f_{1}^{*}(x ; x, t)$ (if there exists) is characterized as the unique point which maximizes (7.4) under the condition (7.3).

Case 1. $\omega_{1}(x)-\omega_{1}(t)+\omega_{1}\left(t_{1}\right)$ is not an integer.

Then we have $\delta=1$. Hence $h_{1}(z)$ has two zeros $\alpha$ and $t$, and therefore the extremal function $f_{1}^{*}(z ; x, t)$ has no zeros on $\bar{A}$. $n_{1}=0$.

(a) $|t|=r^{1 / 2}$ : Then we have $\omega_{1}(t)=\omega_{1}\left(t_{1}\right)$ and $\omega_{1}(x)-\omega_{1}(\alpha)=n_{1}$ and therefore

(b) $r^{1 / 2}<|t|<1$ : We set $D=-\omega_{1}(t)+\omega_{1}\left(t_{1}\right)(0<D<1)$.

(1) $\omega_{1}(x)<1-D$ : we have $n_{1}=0$.

(2) $\omega_{1}(x)>1-D$ : we have $n_{1}=1$. 
(c) $r<|t|<r^{1 / 2}(-1<D<0)$ :

(1) $\omega_{1}(x)<-D$ : we have $n_{1}=-1$.

(2) $\omega_{1}(x)>-D:$ we have $n_{1}=0$.

Case 2. $\omega_{1}(x)-\omega_{1}(t)+\omega_{1}\left(t_{1}\right)$ is an integer:

Then we have $\delta=0$. Hence $h_{1}(z)$ has only one zero point $t$ and therefore the extremal function $f_{1}^{*}(z ; x, t)$ has one zero on $\bar{A}$. Here any zero on the boundary is to be counted with half its multiplicities in this enumeration.

(a) $f_{1}^{*}(z ; x, t)$ has one zero in $A$.

(b) $f_{1}^{*}(z ; x, t)$ has one double zero on $\partial A$.

At first, we note that in the Case 1 the extremal function $f_{1}^{*}(z ; x, t)$ is determined uniquely. Let $\hat{f}_{1}^{*}(z ; x, t)$ be any extremal function which maximizes $f(x)$ in the subfamily of $H_{1}^{t 1}(A)$ satisfying $f(x) \geqq 0$. By the uniqueness of $h_{1}(z)$, from (7.1) we have

$$
\frac{f_{1}^{*}(z ; x, t)}{\hat{f}_{1}^{*}(z ; x, t)}=\frac{\left|f_{1}^{*}(z ; x, t)\right|}{\left|\hat{f}_{1}^{*}(z ; x, t)\right|} \text { on } \partial A
$$

Hence we have $\hat{f}_{1}^{*}(z ; x, t) \equiv f_{1}^{*}(z ; x, t)$.

Now, in the cases (a), (b) (1), (c) (2) in Case 1 and Case 2; (b), $f_{1}^{*}(z ; x, t)^{1 / 2}$ $\left(f_{1}^{*}(x ; x, t)^{1 / 2}>0\right)$ becomes a single-valued analytic function and therefore we have, as we see from the proof of Theorem 6.1,

$$
f_{1}^{*}(z ; x, t)=\frac{R_{t}(z, x)^{2}}{R_{t}(x, x)} .
$$

Hence in these cases, $R_{t}(z, x)$ has no zeros on $\bar{A}$ except for the Case 2; (b), in which $R_{t}(z, x)$ has one simple zero on $\partial A$. Next, in the cases (b) (2) and (c) (1) in Case 1 and Case 2 ; (a), $f_{1}^{*}(z ; x, t)^{1 / 2}$ does not become a single-valued analytic function and therefore

$$
f_{i}^{*}(z ; x, t)=\frac{R_{t}(z, x)^{2}}{R_{t}(x, x)}
$$

is not valid. In particular, from Theorem 6.1 we see that $R_{t}(z, x)$ has one simple zero in $A$ (if there exists, the total number of zeros is at most one as we see from (1.1)).

In summary.

( I ) Case 1; (a), (b) (1) and (c) (2): $R_{t}(z, x)$ has no zeros on $\bar{A}$ and therefore $\mathcal{L}_{t}(\tau, x)=L_{t}(z, x) d z$ has one simple zero in $A$ as we see from (1.1).

(II) Case 2; (b): $R_{t}(z, x)$ has one simple zero on $\partial A$ and therefore $L_{t}(z, x)$ also has the simple zero at the same point on $\partial A$. 
(III) Case 1; (b) (2), (c) (1) and Case 2; (a): $R_{t}(z, x)$ has one simple zero in $A$ and therefore $L_{t}(z, x)$ has no zeros on $\bar{A}$.

It is easy to see that Case (II) in fact may happen.

Finally in connection with the assumption of Theorem 6.1 , we note the existence of the cases such that $R_{t}(z, x)$ has no zeros on $\bar{A}$ and $R_{t}(z, x)^{2 / p}$ $(p>0, p \neq 1,2)$ is not a single-valued analytic function.

\section{REFERENCES}

[1] Ahlfors, L. V., ANd A. Beurling, Conformal invariants and function-theoretic null-sets. Acta Math. 86 (1950), 101-129.

[2] Constantinescu, C., ANd A. Cornea, Ideale Ränder Riemannscher Flächen. Berlin, (1963).

[3] Hejhal, D. A., Classification theory for Hardy classes of analytic functions. Bull. Amer. Math. Soc. 17 (1971), 767-771.

[4] Havinson, S. Ja. Analytic capacity of sets, joint non-triviality of various classes of analytic functions and the Schwarz lemma in arbitrary domains. Amer. Math. Soc. Transl. (2) 43 (1963), 215-266.

[5] Heins, M., Hardy classes on Riemann surfaces. Lecture Notes in Mathematics 98 (1969), $106 \mathrm{pp}$.

[6] Myrberg, P., Über die analytısche Fortsetzung von beschränkten Funktionen. Ann. Acad. Sc1. Fenn. 58 (1949), 7 pp.

[7] Parreau, M., Sur les moyennes des fonctions harmoniques et analytiques et la classification des surfaces de Riemann. Ann. Inst. Fourier 3 (1951), 103-198.

[8] SaItoh, S., The kernel functions of Szegö type on Riemann surfaces. Kōda1 Math. Sem. Rep. 24 (1972), 410-422.

[9] Sario, L., AND K. Oikawa, Capacity functions. Berlin-Heidelberg-New York, Springer (1969), $361 \mathrm{pp}$.

Shibaura Institute of Technology. 